\title{
Plasma plume effects on the conductivity of amorphous-LaAlO3/SrTiO3 interfaces grown by pulsed laser deposition in 02 and $\mathrm{Ar}$
}

Sambri, A.; Christensen, Dennis ; Trier, Felix; Chen, Yunzhong; Amoruso, S.; Pryds, Nini; Bruzzese, R.; Wang, $X$.

Published in:

Applied Physics Letters

Link to article, DOI:

$10.1063 / 1.4727905$

Publication date:

2012

Document Version

Publisher's PDF, also known as Version of record

Link back to DTU Orbit

Citation (APA):

Sambri, A., Christensen, D., Trier, F., Chen, Y., Amoruso, S., Pryds, N., Bruzzese, R., \& Wang, X. (2012). Plasma plume effects on the conductivity of amorphous-LaAlO $/ \mathrm{SrTiO}$ interfaces grown by pulsed laser deposition in $\mathrm{O}_{2}$ and Ar. Applied Physics Letters, 100, 131605.https:/// $\mathrm{doi} .0 \mathrm{rg} / 10.1063 / 1.4727905$

\section{General rights}

Copyright and moral rights for the publications made accessible in the public portal are retained by the authors and/or other copyright owners and it is a condition of accessing publications that users recognise and abide by the legal requirements associated with these rights.

- Users may download and print one copy of any publication from the public portal for the purpose of private study or research.

- You may not further distribute the material or use it for any profit-making activity or commercial gain

- You may freely distribute the URL identifying the publication in the public portal 


\section{AIP Applied Physics \\ Letters}

\section{Plasma plume effects on the conductivity of amorphous-LaAlO3/SrTiO3 interfaces grown by pulsed laser deposition in $\mathrm{O} 2$ and $\mathrm{Ar}$}

A. Sambri, D. V. Cristensen, F. Trier, Y. Z. Chen, S. Amoruso et al.

Citation: Appl. Phys. Lett. 100, 231605 (2012); doi: 10.1063/1.4727905

View online: http://dx.doi.org/10.1063/1.4727905

View Table of Contents: http://apl.aip.org/resource/1/APPLAB/v100/i23

Published by the American Institute of Physics.

\section{Related Articles}

Analysis of thermal conductance of ballistic point contacts

Appl. Phys. Lett. 100, 233109 (2012)

High mobility of the strongly confined hole gas in $\mathrm{AgTaO} / \mathrm{SrTiO} 3$

Appl. Phys. Lett. 100, 201607 (2012)

Memory effects in electrochemically gated metallic point contacts

Appl. Phys. Lett. 100, 203511 (2012)

Quantum point contact with large subband energy spacings

Appl. Phys. Lett. 100, 183502 (2012)

Surface charging, discharging and chemical modification at a sliding contact

J. Appl. Phys. 111, 083501 (2012)

\section{Additional information on Appl. Phys. Lett.}

Journal Homepage: http://apl.aip.org/

Journal Information: http://apl.aip.org/about/about_the_journal

Top downloads: http://apl.aip.org/features/most_downloaded

Information for Authors: http://apl.aip.org/authors

\section{ADVERTISEMENT}

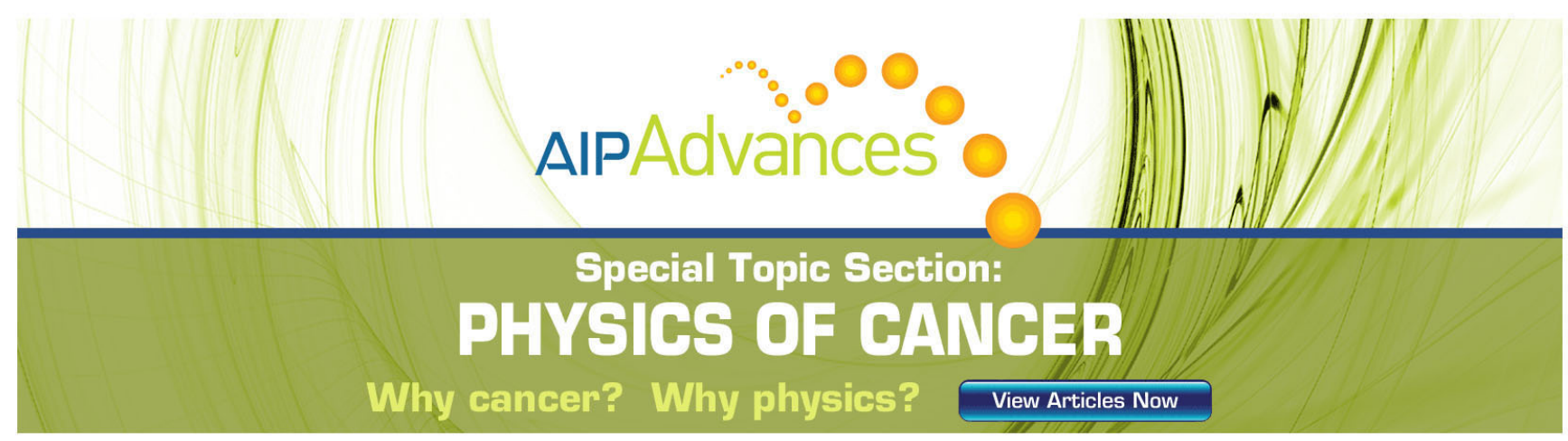




\title{
Plasma plume effects on the conductivity of amorphous-LaAlO ${ }_{3} / \mathrm{SrTiO}_{3}$ interfaces grown by pulsed laser deposition in $\mathrm{O}_{2}$ and $\mathrm{Ar}$
}

\author{
A. Sambri, ${ }^{1}$ D. V. Cristensen, ${ }^{2}$ F. Trier, ${ }^{2}$ Y. Z. Chen, ${ }^{2}$ S. Amoruso, ${ }^{1}$ N. Pryds,,${ }^{2, a)}$ \\ R. Bruzzese, ${ }^{1}$ and X. Wang ${ }^{1}$ \\ ${ }^{1}$ CNR-SPIN and Dipartimento di Scienze Fisiche, Complesso Universitario di Monte Sant' Angelo, \\ Via Cintia, I-80125 Napoli, Italy \\ ${ }^{2}$ Department of Energy Conversion and Storage, Risф Campus, Technical University of Denmark, \\ DK-4000 Roskilde, Denmark
}

(Received 25 April 2012; accepted 16 May 2012; published online 6 June 2012)

\begin{abstract}
Amorphous- $\mathrm{LaAlO}_{3} / \mathrm{SrTiO}_{3}$ interfaces exhibit metallic conductivity similar to those found for the extensively studied crystalline- $\mathrm{LaAlO}_{3} / \mathrm{SrTiO}_{3}$ interfaces. Here, we investigate the conductivity of the amorphous- $\mathrm{LaAlO}_{3} / \mathrm{SrTiO}_{3}$ interfaces grown in different pressures of $\mathrm{O}_{2}$ and $\mathrm{Ar}$ background gases. During the deposition, the $\mathrm{LaAlO}_{3}$ ablation plume is also studied, in situ, by fast photography and space-resolved optical emission spectroscopy. An interesting correlation between interfacial conductivity and kinetic energy of the $\mathrm{Al}$ atoms in the plume is observed: to assure conducting interfaces of amorphous- $\mathrm{LaAlO}_{3} / \mathrm{SrTiO}_{3}$, the kinetic energy of $\mathrm{Al}$ should be higher than $1 \mathrm{eV}$. Our findings add further insights on mechanisms leading to interfacial conductivity in $\mathrm{SrTiO}_{3}$-based oxide heterostructures. C 2012 American Institute of Physics. [http://dx.doi.org/10.1063/1.4727905]
\end{abstract}

Complex oxides heterostructures have been extensively investigated during the last decade due to the variety of intriguing phenomena occurring at their interface. ${ }^{1-3}$ The highmobility electron gas formed at the interface between a polar $\mathrm{LaAlO}_{3}$ (LAO) film and a non-polar $\mathrm{TiO}_{2}$-terminated $\mathrm{SrTiO}_{3}$ (STO) substrate triggered a number of investigations on the transport properties of interfaces based on epitaxial polar oxide thin films. ${ }^{4}$ More recently, it has been demonstrated that metallic interfaces also can be realized by deposition of insulating overlayers of amorphous thin films of LAO, STO, yttria-stabilized zirconia (YSZ), and $\mathrm{CaHfO}_{3}$ on STO single crystal substrates. ${ }^{5,6}$ Interestingly, all these amorphous films were grown by pulsed laser deposition (PLD), and a strong dependence of their conducting properties on the deposition parameters, especially the background oxygen pressure, was observed. ${ }^{5}$ The presence of growth-induced oxygen vacancies in the STO substrate was ascertained as the dominant cause for the interfacial conductivity of these amorphous/crystalline oxide heterostructures. In particular, Chen et al. suggested a mechanism where oxygen vacancies in the STO substrate originate from an outward diffusion of oxygen from the substrate to the oxygen-deficient amorphous overlayer. ${ }^{5}$ This mechanism is driven by surface redox-reactions involving specific chemical species of the ablation plume with high oxygen affinity. The observation of an insulating interface when an amorphous $\mathrm{La}_{7 / 8} \mathrm{Sr}_{1 / 8} \mathrm{MnO}_{3}$ (LSMO) film is deposited on STO supports a scenario in which the plume chemical composition directly affects the conducting properties of the amorphous STO-based heterostructures. ${ }^{5}$ Furthermore, conducting interfaces were only observed when depositing at $\mathrm{O}_{2}$ pressures below $10^{-2} \mathrm{mbar}$, which suggests that the kinetic energy of the ablated species may also play a nontrivial role in the interfacial conductivity. For the film deposition in oxygen, the $\mathrm{O}_{2}$ background pressure determines the kinetic energy of the ablated species, ${ }^{7,8}$ apart from their oxidation degree. ${ }^{8-10}$ The

${ }^{a)}$ Electronic mail: nipr@dtu.dk. individual role of kinetic energy and degree of oxidation is thus a priori unclear. The two effects can, however, be separated by deposition in an inert gas, where the kinetic energy of the ablated species can be modified without resulting in significant oxidation of the plume species.

Here, we report the transport properties of heterostructures produced by deposition of an amorphous LAO ( $a$-LAO) film on a STO substrate at different background pressures of $\mathrm{O}_{2}$ and $\mathrm{Ar}$, while the LAO ablation plume dynamics and composition is determined in situ. Our experimental findings show an interesting correlation between the interfacial conductivity and the kinetic energy of the species impinging on the substrate, thus adding further insights on mechanisms leading to the interfacial conductivity.

The $a$-LAO/STO samples were grown at room temperature (RT), both in $\mathrm{Ar}$ and $\mathrm{O}_{2}$ using a $\mathrm{KrF}$ excimer laser $(\lambda=248 \mathrm{~nm})$ with a repetition rate of $1 \mathrm{~Hz}$ at a fluence of $2.0 \mathrm{~J} \mathrm{~cm}^{-2}$. The target-to-substrate distance was fixed at $4.5 \mathrm{~cm}$. The interfacial conductivity was characterized as described elsewhere. ${ }^{5}$ During deposition, the LAO ablation plume dynamics and composition were investigated both in $\mathrm{Ar}$ and $\mathrm{O}_{2}$ by exploiting fast intensified-charge-coupled device (ICCD) imaging and time- and space-resolved optical emission spectroscopy (OES) ${ }^{7,8}$ While these techniques only monitor optically active species, they provide a reliable description of the influence of various experimental parameters on plume dynamics and composition. This description is also consistent with the results of other different complementary techniques, ${ }^{11}$ e.g., ion probe and time-resolved optical emission spectroscopy, which provide only local information.

Fig. 1 reports the RT sheet conductance $G_{s}$ of $a-\mathrm{LAO} /$ STO samples as a function of the background gas pressure, $p$, for both $\mathrm{Ar}$ and $\mathrm{O}_{2}$. Metallic interfaces are formed for $p \leq 10^{-3}$ mbar in both types of background gases, whereas at $p=10^{-2}$ mbar the $a$-LAO/STO interfaces are insulating for $\mathrm{O}_{2}$ but remains conducting for Ar. Insulating samples in $\mathrm{Ar}$ are only observed for $p \geq 10^{-1} \mathrm{mbar}$. Therefore, the transition 


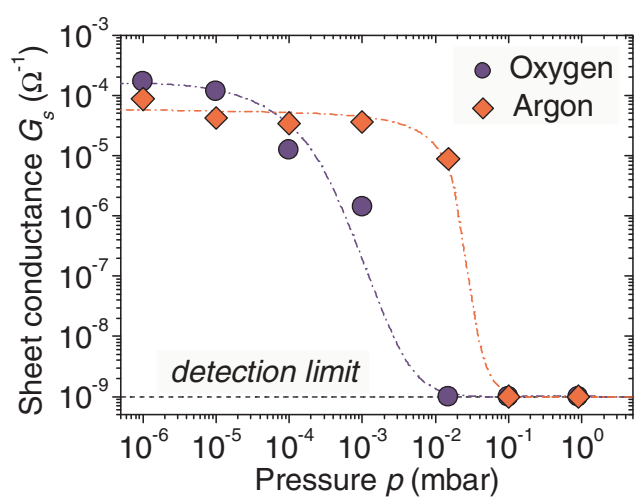

FIG. 1. $a$-LAO/STO interface sheet conductance, $G_{s}$, as a function of the background gas pressure, $p$, for $\mathrm{O}_{2}$ (blue dots) and $\mathrm{Ar}$ (red diamonds). The dash-dot lines are guide for the eye. The dotted line corresponds to the detection limit of the instrument.

from a metallic to an insulating behavior is different for $\mathrm{O}_{2}$ and Ar background. This difference may result from the inherent difference of the kinetic energy or the oxidation degree of the ablated species between the two background gases. Therefore, it can shed light on mechanisms contributing to the interfacial conductivity of $a$-LAO/STO.

In the following, an experimental analysis of the LAO plume in $\mathrm{Ar}$ and $\mathrm{O}_{2}$ is carried out with the aim to get valuable information on its expansion dynamics and composition. Figs. 2(a) and 2(b) (left column) show typical twodimensional (2D) images of the LAO plume emission at a delay of $\tau=3.3 \mu$ s after the laser pulse for deposition in $\mathrm{O}_{2}$ and $\mathrm{Ar}$, respectively, at three different values of the pressure $p$ $\left(10^{-3}, 10^{-2}\right.$, and $\left.10^{-1} \mathrm{mbar}\right)$. The corresponding spatially resolved one-dimensional (1D) spectra of the plume emission, in the range of $\approx 370-630 \mathrm{~nm}$, are also shown in the right columns of Fig. 2. The data at $10^{-3}$ mbar are representative of the plume features registered at lower pressure. The 2D images in Fig. 2 show that the LAO plume propagation dynamics is strongly affected by the background gas pressure. By increasing the pressure from $10^{-3}$ to $10^{-1}$ mbar, the plume becomes more confined, resulting in a progressive reduction of its length and variation of its shape. Both for $\mathrm{Ar}$ and $\mathrm{O}_{2}$, $10^{-2}$ mbar represents the pressure corresponding to the crossover from a free $\left(10^{-3} \mathrm{mbar}\right)$ to a braked $\left(10^{-1} \mathrm{mbar}\right)$ plume expansion. In both gases, the spectra show characteristic emission from $\mathrm{La}$ and $\mathrm{Al}$ neutrals, as well as $\mathrm{LaO}$ diatomic oxides. ${ }^{12,13}$ As expected, the background gas affects the plume composition in different ways.

Considering first the $\mathrm{O}_{2}$ reactive gas, oxidation of the plume occurs as a consequence of gas-phase reactions favored by the effective dissociation of oxygen molecules when the plume follows a shock-wave-like expansion regime at larger pressures. ${ }^{7,14}$ The specific reactivity of the involved cations seems also to influence the LAO plume chemistry. The reaction of La with oxygen is highly exothermic and lanthanum-oxides are effectively produced in electronically excited states, as indeed observed in the spectra of Fig. 2(a). On the contrary, no significant emission from Al-oxides is observed. This is in agreement with our previous reports evidencing the prevalence of $\mathrm{LaO}$ formation during PLD of La-containing complex oxides in oxygen ambient gas. ${ }^{7,8}$
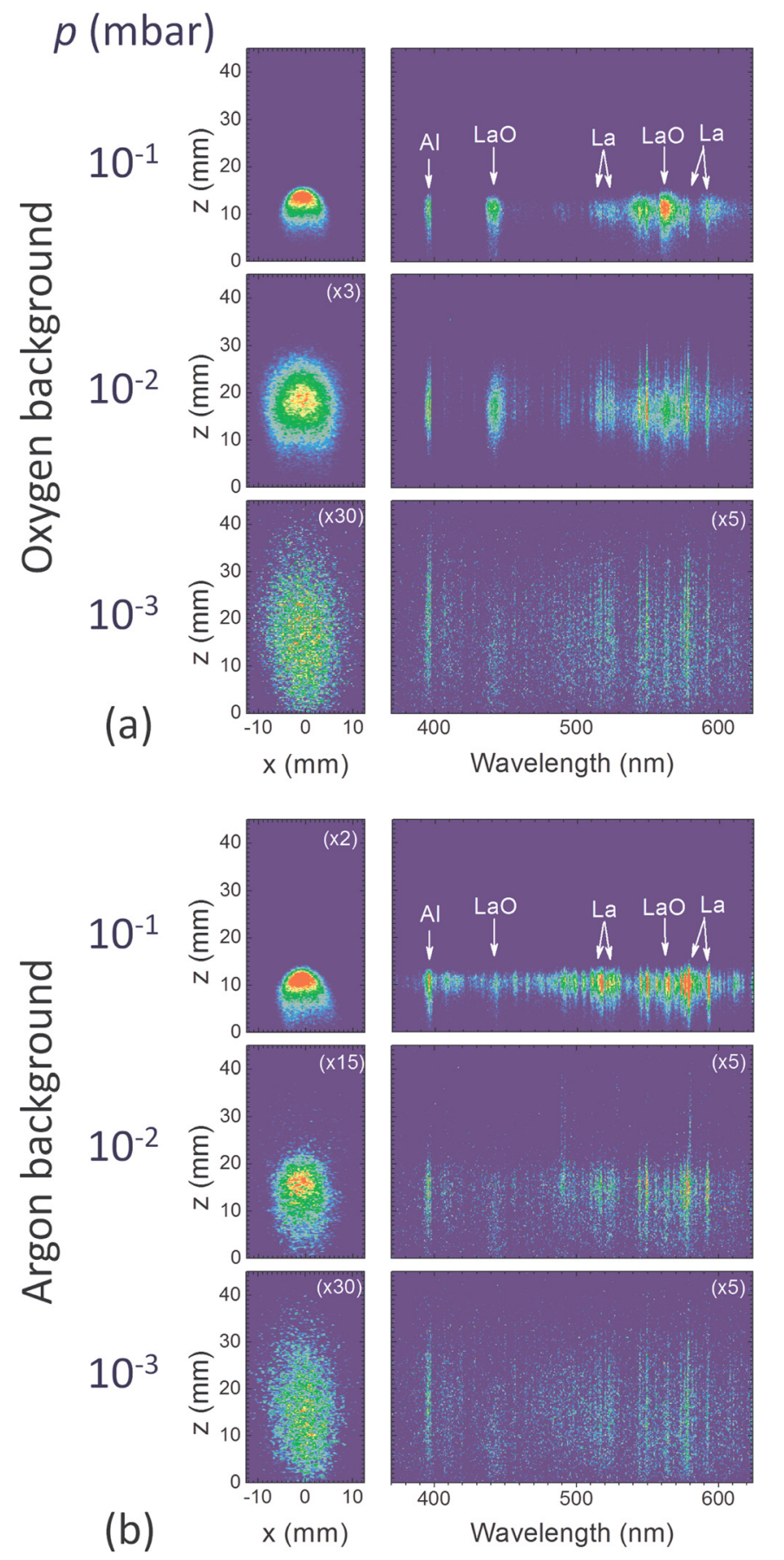

FIG. 2. 2D images (left column) and corresponding 1D spectra (right column) of the LAO plume emission in (a) oxygen and (b) argon background gases, acquired at a delay $\tau=3.3 \mu$ s after the laser pulse, for three different oxygen pressures: $10^{-3} \mathrm{mbar}$ (lower panels), $10^{-2} \mathrm{mbar}$ (central panels), and $10^{-1}$ mbar (upper panels). The plume propagation direction is along the $z$-axis, and $z=0$ marks the position of the target surface, while the $x$-axis is parallel to the target surface. To facilitate the comparison among images and spectra, the intensity is multiplied by an appropriate factor (shown in parenthesis in each panel). The labels in each upper right spectrum show the assignment of the most intense emission features.

The spectra of Fig. 2(a) clearly indicate that the formation of $\mathrm{LaO}$ molecules in the plume is progressively promoted as the $\mathrm{O}_{2}$ pressure increases. At $p=10^{-3}$ mbar the plume emission mainly comes from $\mathrm{Al}$ and $\mathrm{La}$ atomic species. However, the emission intensity of $\mathrm{LaO}$ significantly increases at $p=10^{-2} \mathrm{mbar}$, eventually becoming dominant at $10^{-1}$ mbar, while the emission from La reduces correspondingly. 
TABLE I. Variation of the $\left[\mathrm{LaO}^{*}\right] /\left[\mathrm{La}^{*}\right]$ emission intensity ratio with pressure, $p$, at a distance of $\approx 40 \mathrm{~mm}$ from the target surface. The data at $10^{-4}$ mbar in oxygen is taken as reference and its value is put equal to 1.0. The intensity ratio at $10^{-1} \mathrm{mbar}$ is evaluated at the plume stopping distance of $\approx 20 \mathrm{~mm}$ from the target surface.

\begin{tabular}{lcc}
\hline \hline & \multicolumn{2}{c}{$\left[\mathrm{LaO}^{*}\right] /\left[\mathrm{La}^{*}\right]$} \\
\cline { 2 - 3 }$p$ (mbar) & $\mathrm{O}_{2}$ & $\mathrm{Ar}$ \\
\hline $10^{-4}$ & $1.0^{\mathrm{a}}$ & 0.98 \\
$10^{-3}$ & 1.1 & 0.99 \\
$10^{-2}$ & 3.5 & 1.2 \\
$10^{-1}$ & $5.9^{\mathrm{b}}$ & $1.8^{\mathrm{b}}$ \\
\hline \hline
\end{tabular}

The emission intensity ratio in $\mathrm{O}_{2}$ at $10^{-4}$ mbar was considered as reference and put equal to 1.0 .

${ }^{\mathrm{b}}$ At $10^{-1}$ mbar the plume is stopped. The reported values refer to a distance of $25 \mathrm{~mm}$ from the target surface.

Moreover, $\mathrm{Al}$ emission is always present. It has been shown earlier that the emission intensity ratio between a metal monoxide, $\mathrm{XO}^{*}$, and its metallic element, $\mathrm{X}^{*}$, provides a useful, reliable method to follow the plume oxidation as a result of the interaction with the $\mathrm{O}_{2}$ background gas. ${ }^{9,10}$ Variation of the $\left[\mathrm{LaO}^{*}\right] /\left[\mathrm{La}^{*}\right]$ emission intensity ratio close to the substrate as a function of the pressure $p$ is reported in Table I. The data confirm the progressive enhancement of the oxidized species in the plume as $p$ increases. At $10^{-1}$ mbar the plume propagation is strongly braked and eventually stopped at a distance of $\approx 20 \mathrm{~mm}$ from the target. Therefore, the plume species arrive at the substrate only through diffusion in the background gas. ${ }^{15}$ In this last case, the $\left[\mathrm{LaO}^{*}\right] /\left[\mathrm{La}^{*}\right]$ emission intensity ratio close to the stopping regime was evaluated and reported in Table I.

In the inert Ar gas, a distinctly different variation of the plume spectra with pressure is observed (see Fig. 2(b) and Table I). In this case, the pressure variation of the plume species emission is mainly related to the confining effect of the background gas. At $p=10^{-3}$ mbar of Ar, both the emission spectrum and the $\left[\mathrm{LaO}^{*}\right] /\left[\mathrm{La}^{*}\right]$ emission intensity ratio are very similar to those observed in $\mathrm{O}_{2}$. This is a consequence of the reduced interaction with the background gas. For larger pressures, the plume emission in Ar is mainly due to atomic species, meanwhile the $\mathrm{LaO}$ emission intensity remains comparatively rather weak. Therefore, the $\approx 3 \times$ larger values of the $\left[\mathrm{LaO}^{*}\right] /\left[\mathrm{La}^{*}\right]$ emission intensity observed in $\mathrm{O}_{2}$ is due to the gas-phase oxidation processes occurring during interaction between the plume and the background gas.

During PLD growth of the LAO overlayer in $\mathrm{O}_{2}$ ambient gas, both gas-phase and surface reactions will be important for sample oxidation. Our observations indicate that in $\mathrm{O}_{2}$ the $\mathrm{LaO}$ formation through gas-phase reactions represents a progressively more important channel of oxygen incorporation in the $a$-LAO growing film as the pressure increases, which is indeed not the case for the Ar background. This pivotal difference explains the transition to insulating interfaces observed in $\mathrm{O}_{2}$ already at $p=10^{-2}$ mbar (see in Fig. 1). According to Ref. 5, the interfacial conductivity of the $a$ LAO/STO samples is attributed to the formation of oxygen vacancies in the STO substrate during the deposition. The deposition of a well-oxygenated $a$-LAO overlayer at such a pressure limits the oxygen outward diffusion from the STO substrate. In Ar, the $a$-LAO overlayer is always oxygen deficient. Therefore, the sharp transition from conducting to insulating interfaces, occurring at $p \approx 10^{-1}$ mbar, cannot be ascribed to the degree of oxidation. Instead, the transition clearly indicates that the plume dynamics might play an important role in the formation of oxygen vacancies in the STO substrate and hence for the interface conductivity. Moreover, the observation of a conducting behavior of $a$-LAO/STO interfaces and an insulating behavior of $a$-LSMO/STO, in the same experimental conditions, ${ }^{5}$ suggests that the redox reactions mainly involve $\mathrm{Al}$, rather than $\mathrm{La}$ for the $\mathrm{LAO} /$ STO system, as a consequence of its higher reactivity at the substrate surface. ${ }^{5,16}$

By tracking the front edge of the plume species from a sequence of images such as those in Fig. $2,{ }^{7,8}$ the maximum kinetic energy, $K E_{\max }$, of the species at the substrate position was estimated at each pressure. As discussed above, it is of special concern to follow the pressure variation of $K E_{\max }$ for the Al atoms reaching the substrate. This is shown in Figs. 3(a) and 3(b), where the interfacial sheet conductance $G_{s}$ of the $a$-LAO/STO heterostuctures is also reported in each panel to facilitate the analysis. The kinetic energy of La and $\mathrm{LaO}$ follows a similar trend as a function of the pressure, but it is generally about a factor of 5 higher.

In $\mathrm{Ar}$ [see Fig. 3(a)], the maximal kinetic energy is $\approx 15 \mathrm{eV}$ at $p \leq 10^{-4}$ mbar and reduces slightly as the pressure increases up to $p \approx 10^{-3}$ mbar. Then, a fast decay of $K E_{\max }$ is observed as the pressure increases further. At $p \geq 6 \times 10^{-2} \mathrm{mbar}$, the plume attains a stopping regime over a length shorter than the target-to-substrate distance, and the kinetic energy of the ablated species reduces to the room temperature thermal energy $(1 / 40 \mathrm{eV})$. The sheet conductance, $G_{s}$, follows a rather similar dependence. At $p \approx 1.5 \times 10^{-2}$ mbar, it only reduces slightly with respect to the value observed at lower pressures ( $p \leq 10^{-3}$ mbar), but eventually it turns to insulating as $p$ further increases. In particular, passing from $p \approx 1.5 \times 10^{-2}$ mbar to $p \approx 0.1$ mbar, a transition from a metallic to an insulating

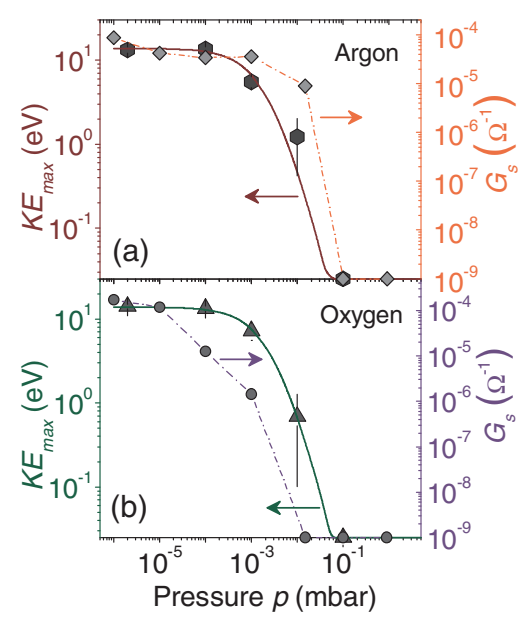

FIG. 3. Maximum kinetic energy of the $\mathrm{Al}$ atoms, $K E_{\max }$, as a function of the background gas pressure, $p$, for $\mathrm{Ar}$ (top panel, hexagons) and $\mathrm{O}_{2}$ (bottom panel, triangles). In each panel, the dependence of sheet conductance, $G_{s}$, on $p$ for the $a$-LAO/STO heterostructures deposited in Ar (top panel, rhombi) and $\mathrm{O}_{2}$ (bottom panel, dots) is also reported. Note that the lower limits for the sheet conductance and the maximum kinetic energy are set to $10^{-9} \Omega^{-1}$ (i.e., the detection limit for the transport measurements) and $1 / 40 \mathrm{eV}$ (i.e., the thermal energy available at room temperature), respectively. 


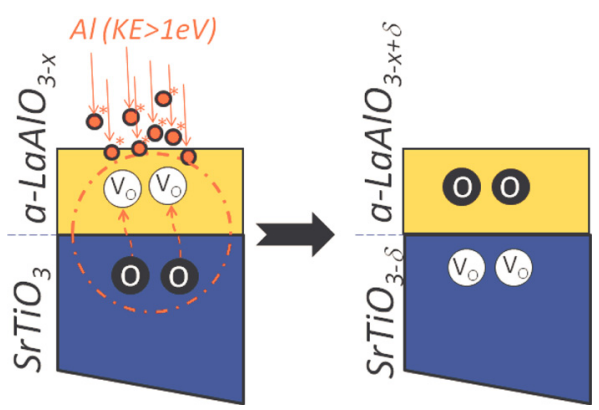

FIG. 4. Schematic representation of the diffusion of oxygen from the STO substrate to the oxygen deficient $a$-LAO film activated by the arrival of chemically active species (e.g., $\mathrm{Al}$ in the case of the LAO plume) during the film growth. A kinetic energy of these reactive species larger than an activation energy threshold of $\approx 1 \mathrm{eV}$ is needed to ensure a conducting interface as a result of forming oxygen vacancies $\left(\mathrm{V}_{\mathrm{O}}\right)$ in $\mathrm{SrTiO}_{3}$.

behavior occurs in correspondence to a reduction of $K E_{\max }$ below $\approx 1 \mathrm{eV}$. This, in turn, suggests that the mechanism of oxygen outward diffusion, from the STO substrate to the oxygendeficient $a$-LAO overlayer, ${ }^{5}$ needs a minimum kinetic energy of the impinging $\mathrm{Al}$ atoms to activate the redox reactions at the interface.

In $\mathrm{O}_{2}$, a rather similar dependence of $K E_{\max }$ with the pressure is observed, but the interface is already insulating at $p \approx 10^{-2}$ mbar, although $\mathrm{O}_{2}$ and Ar are almost equally effective in braking the plume. ${ }^{15}$ This is a consequence of the concurrent effects of a progressive increase of plume oxidation and a simultaneous decrease of $K E_{\text {max }}$. This leads to an enhancement of the oxidation degree of the $a$-LAO overlayer and, at the same time, to a greatly reduced effectiveness of the surface redox reactions. This scenario explains the different values of $\mathrm{O}_{2}$ and $\mathrm{Ar}$ pressure at which insulating interfaces start to be observed.

The observations discussed above suggest that both the oxygen content of the $a$-LAO overlayer and the kinetic energy of the incoming $\mathrm{Al}$ atoms play an important role in the formation of an electron gas at the $a$-LAO/STO interface. Moreover, the different pressure dependence of the heterostructures realized in $\mathrm{Ar}$ and $\mathrm{O}_{2}$ indicates the existence of a mechanism which enhances the kinetics of redox-processes occurring at the STO surface. The effectiveness of such a mechanism becomes hindered when the energy supplied by the impinging $\mathrm{Al}$ atoms is reduced below a threshold value of the order of $\approx 1 \mathrm{eV}$. This estimate is fairly consistent with experimentally determined values of $0.6 \mathrm{eV}$ and $0.8 \mathrm{eV},{ }^{17,18}$ as well as with theoretical predictions based on density functional theory (DFT) calculations, ${ }^{19,20}$ for the activation barriers needed to move neutral oxygen in bulk STO. Therefore our findings may support the following scenario (see Fig. 4): during $R T$ deposition, the reduction of the STO by interfacial redox-reaction may be kinetically inhibited by the mass transport of oxygen. Nevertheless, the impact of fast oxygendeficient species $(\geq 1 \mathrm{eV})$, inherent to an energetic deposition process like PLD, can significantly alter the kinetics of the redox-reactions with the STO substrate, thus favoring the formation of the electron gas at the interface between the amorphous overlayer and the STO substrate.

In conclusion, we studied the transport properties of $a$ LAO/STO heterostuctures grown by PLD at $R T$ as well as the properties of the ablation plume dynamics and composition, in different background pressures of $\mathrm{O}_{2}$ and Ar. Our results show a striking correlation between kinetic energy of the Al species impinging on the substrate and interface conductivity of LAO/STO. This adds further insights on mechanisms leading to the interfacial conductivity observed in complex oxide heterostructures based on amorphous overlayers on STO. ${ }^{5}$ Our experimental findings support a mechanism in which the formation of oxygen vacancies is favored by redox-reactions at the interface triggered by the arrival of energetic plume species with high oxygen-affinity.

We thank J. Schou, E. Stamate, C. Aruta, and E. Di Gennaro for their valuable help. The research has received funding from European Union Seventh Framework Programme (FP7/ 2007-2013) under Grant Agreement No. 264098-MAMA.

${ }^{1}$ J. Mannhart and D. G. Schlom, Science 327, 1607 (2010).

${ }^{2}$ P. Zubko, S. Gariglio, M. Gabay, P. Ghosez, and J.-M. Triscone, Annu. Rev. Condens. Matter Phys. 2, 141 (2011).

${ }^{3}$ M. Gibert, P. Zubko, R. Scherwitzl, J. Iniguez, and J.-M. Triscone, Nat. Mater. 11, 195 (2012).

${ }^{4}$ A. Ohtomo and H. Y. Hwang, Nature (London) 427, 423-426 (2004).

${ }^{5}$ Y. Z. Chen, N. Pryds, J. E. Kleibeuker, G. Koster, J. R. Sun, E. Stamate, B. G. Shen, G. Rijnders, and S. Linderoth, NanoLett. 11, 3774 (2011).

${ }^{6}$ K. Shibuya, T. Ohnishi, M. Lippmaa, and M. Oshima, Appl. Phys. Lett. 9, 232106 (2007).

${ }^{7}$ S. Amoruso, C. Aruta, R. Bruzzese, D. Maccariello, L. Maritato, F. M. Granozio, P. Orgiani, U. S. di Uccio, and X. Wang, J. Appl. Phys. 108, 043302 (2010).

${ }^{8}$ C. Aruta, S. Amoruso, R. Bruzzese, X. Wang, D. Maccariello, F. Miletto Granozio, and U. S. di Uccio, Appl. Phys. Lett. 97, 252105 (2010).

${ }^{9}$ R. C. Dye, R. E. Muenchausen, and N. S. Nogar, Chem. Phys. Lett. 181, 531 (1991).

${ }^{10}$ P. Lecoeur, A. Gupta, P. R. Duncombe, G. Q. Gong, and G. Xiao, J. Appl. Phys. 80, 513 (1996).

${ }^{11}$ S. Amoruso, R. Bruzzese, R. Velotta, N. Spinelli, M. Vitiello, and X. Wang, Appl. Surf. Sci. 248, 45 (2005).

${ }^{12}$ W. C. Martin, J. R. Fuhr, D. E. Kelleher, A. Musgrove, J. Sugar, W. L. Wiese, P. J. Mohr, and K. Olsen, NIST Atomic Spectra Database, Version 2.0, National Institute of Standards and Technology, Gaithersburg, MD, 1999 .

${ }^{13}$ R. W. B. Pearse and A. G. Gaydon, The Identification of Molecular Spectra, 4th ed. (Chapman and Hall, London, 1984).

${ }^{14}$ A. Camposeo, F. Cervelli, F. Fuso, M. Allegrini, and E. Arimondo, Appl. Phys. Lett. 78, 2402 (2001).

${ }^{15}$ S. Amoruso, B. Toftmann, and J. Schou, Phys. Rev. E 69, 056403 (2004).

${ }^{16} \mathrm{Q}$. Fu and T. Wagner, J. Phys. Chem. 109, 11697 (2005).

${ }^{17}$ F. Cordero, Phys. Rev. B 76, 172106 (2007).

${ }^{18}$ A. Kalabukhov, R. Gunnarsson, J. Börjesson, E. Olsson, T. Claeson, and D. Winkler, Phys. Rev. B 75, 121404(R) (2007).

${ }^{19}$ M. Lontsi-Fomena, A. Villesuzanne, J.-P. Doumerc, C. Frayret, and M. Pouchard, Comput. Mater. Sci. 44, 53 (2008).

${ }^{20}$ D. V. Christensen and F. Trier, Master's thesis, University of Copenaghen, 2012. 